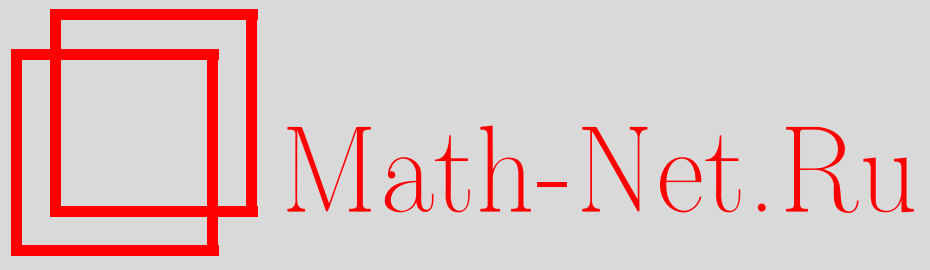

Г. Мерзон, Формула Пика и тающий лед, Квант, 2018, номер 9, 3637

DOI: https://doi.org/10.4213/kvant20180905

Использование Общероссийского математического портала Math-Net.Ru подразумевает, что вы прочитали и согласны с пользовательским соглашением

http://www . mathnet.ru/rus/agreement

Параметры загрузки:

IP: 3.95 .254 .165

26 апреля 2023 г., 14:28:03 


\section{Формула Пика и тающий лед}

\section{Г.MEP3OH}

\section{Формула Пика}

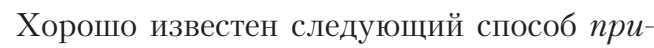
ближенного вычисления площади фигуры на клетчатой бумаге: к числу клеток, полно-

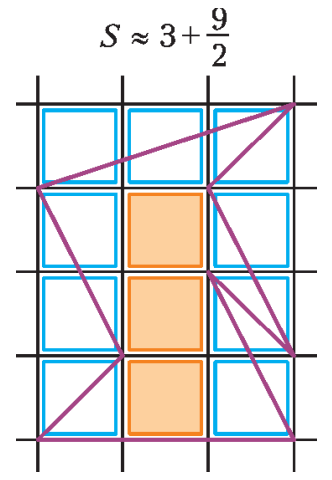

Pис. 1 стью занятых фигурой, надо прибавить половину от числа клеток, занятых частично (рис.1). Но замечательно, что небольшой модификацией этот нехитрый рецепт можно превратить в точную формулу для площади многоугольника.

Формула Пика. Плошадь многоугольника с вершинами в узлах сетки может быть найдена по формуле

$$
S=i+\frac{b}{2}-1
$$

где $i$ - число узлов сетки (строго) внутри многоугольника, $b$ - число узлов сетки на его границе.

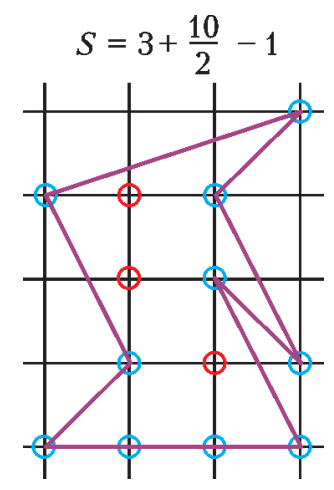

PUC. 2
Пример использования формулы Пика приведен на рисунке 2.

Из этой формулы видно, кстати, что площадь многоугольника с вершинами в узлах сетки всегда является целой или полуцелой (что априори не очевидно даже для треугольников: представьте себе вычисление этой площади по формуле Герона...).
Известны разные доказательства формулы Пика (см., например, [1], [2]). В большинстве из них сначала рассматривают случай треугольника, а затем сводят к нему формулу Пика для произвольного многоугольника.

Мы обсудим мысленный эксперимент, который предложил Кристиан Блаттер ([3]; см. также изложение в [4]), непосредственно доказывающий формулу Пика для любого многоугольника. Но сначала перепишем правую часть формулы немного в другом виде.

\section{Веса узлов}

Площадь фигуры можно оценить приблизительно, просто посчитав количество узлов, которые эта фигура покрывает. Чтобы посчитать точнее, посмотрим на узлы чуть внимательнее: узлы строго внутри многоугольника учитываются с весом 1 , а узлы на границе - с весом $1 / 2$ («лежат внутри наполовину»). Естественно сделать следующий шаг и начать по-разному учитывать вершины многоугольника и узлы сетки на его сторонах.

Нарисуем вокруг каждого узла сетки по кругу маленького радиуса и посчитаем каждый узел с таким весом, какая часть этого круга лежит внутри нашего многоугольника (т.е. точка вне многоугольника получает вес 0 , точка строго внутри - вес 1, «обычная» точка на стороне - вес $1 / 2$, вершина угла $\alpha$ - вес $\alpha /(2 \pi))$.

Теорема. Площадь многоугольника с вершинами в узлах сетки равна количеству занятых им узлов сетки, посчитанных с указанными выше весами.

Утверждение этой теоремы равносильно формуле Пика. Действительно, вес каждой из $i$ внутренних вершин равен 1. А вес каждой из $b$ вершин на границе отличается от $1 / 2$ на внешний угол в этой точке, де-

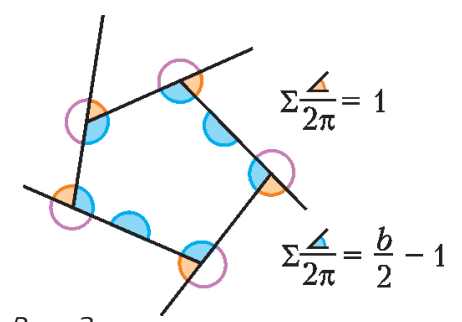

Pис. 3 
ленный на $2 \pi$. Так как сумма всех внешних углов многоугольника равна $2 \pi$, сумма весов внешних вершин равна $b / 2-1$ (рис.3).

Такая формулировка формулы Пика в определенном смысле более естественна. И в такой форме теорема буквально верна и для «многоугольников с дырками» (тогда как обычная формула Пика требует некоторой модификации). С другой стороны, в этой формулировке перестала быть видна полуцелочисленность площади...

\section{Тающий лед}

Перейдем к доказательству теоремы.

Представим себе, что в каждом узле сетки стоит по тонкому цилиндрическому столби-

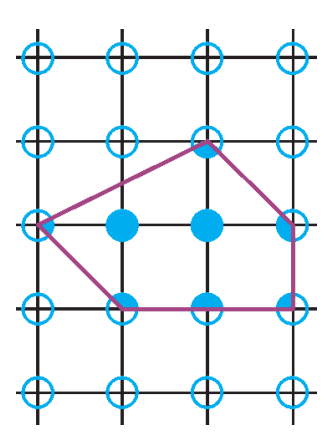

Pис. 4 ку льда. Вес (точнее - масса) каждого столбика равен 1; радиус у всех столбиков одинаковый и достаточно маленький, чтобы столбик в узле, не лежащем на стороне, не пересекал эту сторону. Тогда общий вес льда внутри нашего многоугольника как раз равен сумме весов узлов (рис.4).

Растопим теперь весь лед. Вода растечется равномерно по плоскости. У тверждение теоремы состоит в том, что количество воды внутри многоугольника (площадь) в точности равно количеству льда, который находился внутри многоугольника (сумма весов).

Чтобы не думать, как именно течет и перемешивается бесконечное количество воды на бесконечной плоскости, можно по-

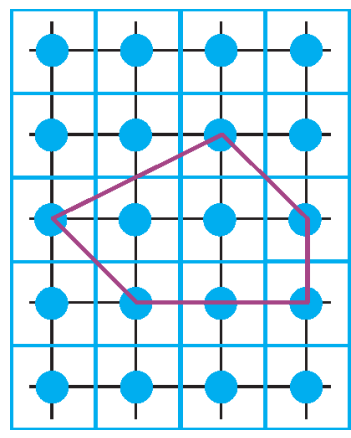

Рис. 5 строить вокруг каждого из столбиков льда по забору в виде единичного квадратика (рис.5) и считать, что каждый столбик растекается по своему квадрату одинаковым и центральносимметричным образом.

Посмотрим на по- ток воды через какуюнибудь сторону многоугольника. Исходная расстановка столбиков льда симметрична относительно середины этой сторонь. Значит, симметричны и потоки воды при таянии льда

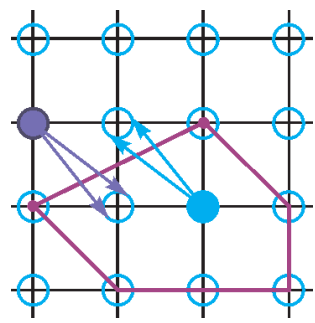

Pис. 6 (рис.6). В частности, через каждую сторону многоугольника втекло столько же воды, сколько вытекло!

Теорема доказана.

\section{Обобщения}

Точно такое же рассуждение показывает, что объем многогранника (с вершинами в узлах сетки) равен сумме весов занимаемых им узлов, если каждая грань многогранника имеет центр симметрии.

Последнее ограничение существенно: если взять какой-нибудь тетраэдр, то его объем обычно не равен сумме вкладов узлов (можете убедиться в этом, например, для правильного тетраэдра, вписанного в единичный куб).

Тем не менее, рассматривая вместо сумм весов некоторые многочлены («solid-angle polynomials»), можно получить формулы для объемов многогранников любой размерности. В частности, можно получить формулу для объема многогранника в трехмерном пространстве, если учитывать кроме узлов исходной сетки узлы вдвое более мелкой сетки.

Другая возможность состоит в том, чтобы по-другому выбирать веса. На этом пути тоже можно получить замечательные формулы для объемов многогранников. Но об этом поговорим в другой раз.

\section{Литература}

1. Н.Васильев. Вокруг формулы Пика. «Квант», 1974, № 12 .

2. А.Кушниренко. Целые точки в многоугольниках и многогранниках. - «Квант», 1977, №4.

3. C.Blatter. Another Proof of Pick's Area Theorem. - Mathematics Magazine, 1997, 70.

4. S.Tabachnikov. Proofs (not) from The Book. - Mathematical Intelligencer, 2014, 36 (2). 